\title{
Philosophiques
}

\section{Richard Gervais : Dialectique et totalitarisme, Montréal, Hurtubise HMH, coll. " Brèches », 1990, 231 p.}

\section{Serge Cantin}

Volume 18, numéro 2, automne 1991

URI : https://id.erudit.org/iderudit/027158ar

DOI : https://doi.org/10.7202/027158ar

Aller au sommaire du numéro

Éditeur(s)

Société de philosophie du Québec

ISSN

0316-2923 (imprimé)

1492-1391 (numérique)

Découvrir la revue

Citer cet article

Cantin, S. (1991). Richard Gervais : Dialectique et totalitarisme, Montréal,

Hurtubise HMH, coll. « Brèches ", 1990, 231 p. Philosophiques, 18(2), 171-166.

https://doi.org/10.7202/027158ar d'utilisation que vous pouvez consulter en ligne.

https://apropos.erudit.org/fr/usagers/politique-dutilisation/ 
RICHARD GERVAIS: Dialectique et totalitarisme, Montréal, Hurtubise HMH, coll. "Brèches », 1990, 231 p.

\author{
par Serge Cantin
}

Tandis qu'au firmament de la pensée occidentale achève de s'éteindre l'astre (...ou le désastre) du marxisme, dont rien ne semble vouloir subsister de l'éclat passé qu'un amas de débris livresques, voici que par un curieux anachronisme la pensée québécoise accouche de son premier véritable essai sur le marxisme. Doit-on s'en réjouir ou s'en affliger, s'en féliciter ou s'en inquiéter, se dire qu'après tout mieux vaut tard que jamais ou, plutôt, déplorer une fois de plus l'excentricité du champ intellectuel québécois qui voue les chercheurs d'ici à la contemplation d'étoiles mortes?

Il ne fait aucun doute que, sous son aspect théorique ou épistémologique, le marxisme n'aura été au Québec qu'un produit $\mathrm{d}^{\prime}$ importation, dont les diverses étiquettes (française surtout) portent au dos la marque de notre aliénation intellectuelle. Prenons garde toutefois que cette évidence ne vienne d'abord remplir une fonction défensive, analogue à celle que la psychanalyse attribue au déplacement. Il se pourrait en effet que l'empressement que l'on met aujourd'hui à localiser dans le passé (même récent) notre colonisation mentale, à l'objectiver, relève d'une tentative pour échapper à la prise de conscience du fait que nous sommes toujours mentalement colonisés. Ce qui expliquerait peut-être, en partie du moins, une réticence assez générale à réfléchir sur notre "marxisme des années soixante " ${ }^{1}-$ comme si la pensée québécoise appréhendait de reconnaître sous la figure d'un marxisme essentiellement idéologique, mimétique et compensatoire, la pérennité de certains de ses vieux blocages.

Pourquoi le milieu intellectuel québécois fut, au sortir de la révolution tranquille, un terrain si propice à la propagation d'une scolastique plus sectaire encore que celle dont il venait à peine de briser le carcan, c'est là une question qu'on ne règlera ni par un boutade (ah! ce que nous avons pu être naïs de croire au grand soir), ni en arguant de l'indigence théorique du marxisme québécois pour faire comme si celui-ci n'avait jamais existé. Car il y a eu, en 
dépit ou peut-être à cause de cette pauvreté théorique, un marxisme québécois, c'est-à-dire une façon typiquement québécoise de se vouloir marxiste, révélatrice de ce que nous sommes et dont l'analyse contribuerait sans doute à lever un peu le voile sur ce que cache la longue fuite en avant de la pensée québécoise. "Alors que la réflexion, dit Richard Gervais, réclamait - et réclame encore - le bilan de cette expérience historique durable et quand même marquante, on dirait que ceux qui font métier d'intelligence sont passés du marxisme au nonmarxisme en perdant la mémoire au lieu de gagner en lucidité. En ce sens, n'y a-t-il pas une certaine imposture à prétendre vivre aujourd'hui dans une époque de marxisme dépassé ? Qui prétendra que cette étonnante aventure n'a plus rien à nous apprendre? qu'est épuisé son bilan philosophique? On a tourné la page d'on ne sait quel livre ...» (pp. 13-14).

On pourra regretter que, dans un livre publié au Québec, aucune place ne soit faite à la forme spécifiquement québécoise d'une telle imposture, l'A. préférant plutôt s'en tenir (mais c'est là son droit le plus strict) à la déconstruction du "mythe dialecticien", mythe dont il subodore la survivance dans l'insistance même que mettent certains à en proclamer le dépassement. Mais puisque ce prétendu dépassement n'est pas moins suspect ici qu'il ne l'est par exemple en France (le dépassement ne constitue-t-il pas le mouvement même de la dialectique?), le livre de $M$. Gervais devrait, du moins en principe, mériter l'attention de ceux et celles (tout bien vérifié, pas mal nombreux) qui, à une époque encore proche et en divers lieux de la société québécoise, s'abandonnèrent aux vertus magiques de la dialectique. En principe seulement, car il est prévisible que " peu enclins à prendre la mesure de leur marxisme d'hier » (p. 13) la plupart tourneront le dos à un ouvrage qui les obligerait à revenir en arrière et à s'interroger sur la part qu'ils prirent dans ce que l'A. n'hésite pas à appeler la "terreur intellectuelle »².

Époque de terreur intellectuelle et donc aussi de censure, où le marxisme totalitaire, quand on ne poussait pas l'aveuglement jusqu'à en nier l'existence, se trouvait le plus souvent réduit à un malencontreux accident de l'histoire, accident qu'il fallait être de droite ou (ce qui, à l'époque, revenait au même) de mauvaise foi pour seulement imaginer que Marx pût en être en quelque façon responsable.

C'est cette censure - toujours, quoique moins ouvertement, à l'oeuvre dans la pensée actuelle - que veut lever M. Gervais en s'attaquant à "l'esquive, qui consiste à nier tout rapport essentiel entre le totalitarisme communiste et la pensée marxienne" (p. 12). Esquive car s'il est une chose évidente selon l'A., une chose que l'on aurait dû apercevoir depuis bien longtemps si le désir de ne pas la voir, et l'espoir correspondant, n'avaient été les plus forts, c'est bien la " compromission " de Marx dans "l'entreprise totalitaire " (p. 13), compromission dont la dialectique hégéliano-marxiste constituerait en elle-même la preuve irréfutable. C'est précisément là ce que l'A. 
va s'employer à démontrer: que " pensée marxienne et totalitarisme marxiste ne sont pas deux choses séparées l'une de l'autre - comme le voudraient les "marxistes critiques" et les "marxologues" (p. 22), mais qu'ils représentent plutôt les deux pôles, philosophique et politique, d'une même illusion dialecticienne.

La démonstration de cette thèse parcourt trois étapes. D'abord, après avoir identifié dans son Introduction les différentes façons dont on s'y est pris jusqu'à maintenant pour éluder la question du rapport entre marxisme et totalitarisme, et laissé de côté les deux plus grossières (i.e. "l'apologie systématique " propre à "une certaine orthodoxie de gauche" et "la détraction systématique de la part de leurs homologues de "droite" ", p. 14 ), l'A. va procéder (cf. Le marxisme comme problème, pp. 17-57) à un examen approfondi des deux manoeuvres les plus astucieuses: celle du marxisme non orthodoxe et celle de la marxologie, qui ne seraient l'une et l'autre, à tout prendre, que des "esquives" commandées par la volonté d'absoudre Marx de toute faute totalitaire.

Face à cet escamotage de la question et, plus généralement, à "l'ignorance philosophique [...] à propos du totalitarisme » (p. 73), l'A. s'efforce ensuite, dans la seconde partie de son ouvrage (Totalitarisme, pp. 59-100), de dégager les caractéristiques essentielles et de cerner l'enjeu philosophique du phénomène totalitaire. Rapportant celui-ci à "la visée de l'unité sociale parfaite" (p. 99) qui hante l'idéal communiste - visée qui a pour corollaire la «contestation radicale de la différenciation de la société en sphères politique et civile» (p. 100) - I'A. retrace, dans la dernière partie (Dialectique, pp. 101-201), la présence de cette obsession antipolitique de l'Un dans l'oeuvre de Marx, plus précisément dans l'Aufhebung de la dialectique de Hegel, dans cette "dialectique matérialiste " en quoi consiste "le véritable utopisme de Marx».

En conclusion (pp. 203-206), l'A. reconnaît dans certains courants de la pensée contemporaine "la perpétuation de cette illusion dialecticienne " qui fut responsable de "l'utopique suppression du caractère formel des droits" - ces droits formels tant dénoncés par Marx et dans lesquels l'A semble voir, pour sa part, le meilleur rempart contre le système totalitaire.

L'ouvrage de M. Gervais représente sans nul doute un apport original dans un domaine singulièrement négligé par les chercheurs d'ici, alors que la discussion du rapport entre la théorie marxienne (ou marxiste) et la pratique marxiste totalitaire se poursuit presque sans interrruption depuis une bonne trentaine d'années en Europe et aux États-Unis. Sans être toujours nouveaux, les éléments que $\mathrm{l}^{\prime} \mathrm{A}$. apporte à ce contentieux - qui reste ouvert - s'articulent en une analyse rigoureuse du contenu philosophique et de la signification 
politique de cette raison dialectique trop souvent invoquée en méconnaissance de cause ou encore comme alibi en certains quartiers intellectuels. Cela dit, la réflexion de $\mathrm{M}$. Gervais nous semble présenter certains écueils qu'il importe de signaler.

D’abord une difficulté de méthode. Dans la seconde partie, Totalitarisme, l'A. souligne à bon droit l'insuffisance de la méthode empiriste et descriptive qui ne fournit que des définitions statiques du phénomène totalitaire. Mais on peut se demander si la méthode qu'il lui oppose, bien qu'elle mette l'accent sur certains traits organiques et dynamiques de ce phénomène, est en mesure de poser correctement les termes du débat sur la nature du totalitarisme. M. Gervais fait reposer les conditions méthodologiques d'un tel débat sur "l'alternative fondamentale " (p. 63) entre raison cartésienne (critique huxleyenne) et raison d'État (critique orwellienne). Cette alternative nous paraît plutôt abstraite, de même que la décision de l'A. de ne considérer le totalitarisme que comme un "avatar" de la raison d'État - décision qui implique non seulement que cette forme politique typiquement moderne qu'est le totalitarisme s'expliquerait indépendamment de la raison cartésienne et technicienne (et de l'aliénation au monde qu'elle présuppose), mais que celle-ci pourrait à la limite être utilisée efficacement contre le totalitarisme. Voilà qui est pour le moins discutable. À recourir à une telle alternative, on risque à notre avis de laisser échapper l'enjeu capital du marxisme qui se situe justement sur le plan de la synthèse nouvelle que Marx opère entre raison cartésienne et raison d'État, c'est-à-dire entre les impératifs typiquement modernes de rationalisation et de production du monde et de la société, d'une part, et, d'autre part, le concept traditionnel de gouvernement qui, depuis Platon, repose sur le désir d'instaurer un ordre permanent dans les affaires humaines, un État définitif où serait surmontée l'imprévisibilité inhérente à l'action humaine.

Cette fausse alternative méthodologique se répercute à coup sûr sur le plan de la problématique mise en place par $\mathrm{M}$. Gervais pour élucider le rapport entre le marxisme et le totalitarisme. Il a tout à fait raison de dire que: “Les marxistes se livrent à la politique parce qu'ils détestent la politique. Pour eux, l'action politique vise "dialectiquement" à délivrer le monde de la politique» (p. 62). Mais de là à prétendre que le concept hégéliano-marxien de dialectique puisse, à lui seul, fournir un principe d'explication du totalitarisme marxiste ( Prise globalement, notre étude, qui focalise sur la dialectique la discussion des rapports du marxisme et du totalitarisme..., p. 134), il n'y a qu'un pas, que l'A. franchit trop vite. Car si tel était le cas, si le totalitarisme marxiste n'était que la résultante de la dialectique hégéliano-marxienne, il deviendrait alors extrêmement difficile de comprendre pourquoi la dialectique platonicienne, elle, en dépit des efforts très sérieux de Platon pour la rendre politiquement opératoire - et ce dans le même dessein que Marx, à savoir la destruction du politique en tant que domaine d'activité spécifique 
- n'a pas marché dans le réel, pourquoi elle est restée à l'état d'utopie, tandis que "l'utopisme dialectique " de Marx a fonctionné, a trouvé un écho (et quel écho!) dans la réalité historique. Et cette objection ne saurait être levée simplement en invoquant l'incommensurabilité des deux dialectiques, la platonicienne et l'hégéliano-marxienne, car ce que nous force à penser la réussite historique du marxisme c'est non pas tant "la liaison entre l'intelligibilté dialectique et le modèle totalitaire " (p. 134), au sens d'une correspondance formelle entre deux formations discursives (entre un certain discours philosophique et un certain discours politique), que ce qui, au sein de la culture moderne mème, fonde la possibilité de cette liaison et a permis et justifié ce transfert réussi de la dialectique dans la pratique politique. Tout se passe comme si pour $\mathrm{I}^{\prime} \mathrm{A}$. la condition de ce transfert résidait dans la raison dialectique elle-même (par exemple: "La raison dialectique est le passeport philosophique du négativisme social", p. 200). Or il s'en faut de beaucoup, selon nous, que la dialectique, qui n'est après tout qu'une idée, renferme en elle-même un tel pouvoir, une telle efficace historique. Comme dit Hannah Arendt, "ce ne sont pas les idées, ce sont les événements qui changent le monde [...] et ce serait se leurrer et faire injure à la gravité des problèmes de l'époque moderne que de les considérer uniquement du point de vue de l'évolution des idées $»^{3}$.

En privilégiant ce point de vue, il était quasiment fatal que l'A. ne vit dans le totalitarisme que le résultat d'une illusion spéculative dont (sans que l'on sache d'ailleurs trop comment elle est parvenue à se transposer dans la réalité historique) il suffirait de dénoncer critiquement l'usage pour que la philosophie en soit quitte avec le phénomène totalitaire.

En somme, ce qui manque le plus au livre de M. Gervais, c'est une réflexion sur l'histoire, c'est-à-dire sur un certain nombre d'événements et de transformations historiques qui, à l'époque moderne, vont préparer le terrain à l'avènement du totalitarisme et peut-être rendre son recours inévitable. Il eût fallu insister, nous semble-t-il, sur ce qui, dans la culture moderne, affirme l'immanence de la vérité à l'histoire, sur ce qui au sein de la société, dans le travail et dans l'industrie notamment, à la fois appelle et vérifie l'hypothèse anthropologique radicale du matérialisme dialectique. Demeure inexploré par l'A. ce que lui-même du reste appelle, à la toute fin de son dernier chapitre, ce "contexte du "désenchantement du monde" ", ce "contexte historique de désacralisation" qui "explique probablement la reformulation hégélienne de la pensée dialectique " (p. 200).

$C^{\prime}$ est cette lacune qui trace la limite d'un ouvrage par ailleurs intéressant, fort bien écrit et, répétons-le, pertinent. Un ouvrage dont nous ne pouvons en définitive que recommander la lecture. 


\section{NOTES}

1. Pour reprendre le titre de l'excellent ouvrage de Maurice Lagueux, Le marxisme des années soixante, Montréal, Hurtubise $\mathrm{HMH}$, coll. "Brèches", 1982. À notre avis, ce livre est le seul autre ouvrage qui pourrait revendiquer le titre de premier véritable essai paru au Québec sur le marxisme, n'était que la problématique de $\mathrm{M}$. Lagueux ne vise qu'un certain marxisme, celui d'Althusser, qu'elle examine par surcroît dans une perspective strictement épistémologique.

2. “Il suffit de se remémorer l'atmosphère qui régnait dans nos milieux universitaires au cours des années 1970 pour se convaincre de ce qui paraît aujourd'hui incroyable. Le marxisme exerçait une sorte de terreur intellectuelle » (p. 20). Maurice Lagueux a, de son côté, fort bien décrit cette atmosphère qui ne se limitait pas aux enceintes universitaires: "C'était l'époque où au Québec un professeur de Cégep pouvait avec fierté et assurance annoncer à ses étudiants qu'il allait les initier à la "science marxiste", l'époque où les thèmes traditionnels de notre histoire nationale ne se profilaient plus qu'assez timidement derrière l'analyse souvent un peu brouillonne de la lutte des classes et, assez paradoxalement, l'époque où, plus que jamais, on s'est inquiété de voir notre système d'éducation outrageusement orienté vers la reproduction des classes dominantes » (op. cit., p. 20).

3. Condition de l'homme moderne, Paris, Calmann-Lévy, 1983, pp. 307 et 353 . 